\title{
Prediction of Single Nucleotide Polymorphisms in TNFSF4 and Confirmation of Its Relationship with AITD and SLE: Bioinformatics Approach
}

\author{
Sevgi Kalkanli Tas ${ }^{1,}$, , Eylem Cagiltay ${ }^{2}$, Duygu Kirkik ${ }^{3}$, Nevin Kalkanli \\ ${ }^{1}$ Department of Immunology, Faculty of Medicine, University of Health Sciences, Istanbul, Turkey \\ ${ }^{2}$ Department of Endocrinology, Sultan Abdulhamid Han Training \& Research Hospital, Istanbul, Turkey \\ ${ }^{3}$ Department of Medical Biology, Faculty of Medicine, University of Health Sciences, Istanbul Turkey \\ ${ }^{4}$ Department of Dermatology, Ozel Diyarlife Hospital, Diyarbakir, Turkey
}

\section{Email address:}

skalkanlitas@gmail.com (S. K. Tas), eylem723@yahoo.com (E. Cagiltay),dygkirkik@gmail.com (D. Kirkik), nevinkalkanli@hotmail.com (N. Kalkanli)

${ }^{*}$ Corresponding author

\section{To cite this article:}

Sevgi Kalkanli Tas, Eylem Cagiltay, Duygu Kirkik, Nevin Kalkanli. Prediction of Single Nucleotide Polymorphisms in TNFSF4 and Confirmation of Its Relationship with AITD and SLE: Bioinformatics Approach. Computational Biology and Bioinformatics.

Vol. 7, No. 1, 2019, pp. 5-10. doi: 10.11648/j.cbb.20190701.12

Received: June 27, 2019; Accepted: July 17, 2019; Published: July 30, 2019

\begin{abstract}
Tumor Necrosis Factor Ligand Superfamily Member4 (TNFSF4) has a huge family of physically homologous transmembrane proteins that regulate various functions in responding cells containing proliferation, differentiation, apoptosis, and inflammatory gene expression. TNFSF4 can play significant role in inflammatory diseases that its polymorphisms of the TNFSF4 gene are mostly related with Sjogren's syndrome, and systemic lupus erythematosus (SLE). The aim of this study is to investigate the genetic variations that may alter the expression, function and role of the TNFSF4 by using in silico methods. Single Nucleotide Polymorphisms (SNPs) on TNFSF4 are analyzed by GeneMania, SIFT, PolyPhen2, UTRscan programme, U.S. National Library of Medicine Database, ClinVar. 37 variants of TNFSF4 were found that among these 9 missense, 8 coding synonymous, 1 coding, 1 splice-3, 1 UTR-3, 11 intron, 5 UTR-5 variants. Moreover, two of them SNPs that these are rs 199835957, rs372063551 were detected probably damaging by PolyPhen2 and they should be noted that vital candidates in causing diseases related to TNFSF4 and they were identified missense variants that weren't reported in ClinVar. In the future, genes of TNFSF4, TNFSFR and CD40 may be studied on polymorphisms with experimental analysis in order to contribute to science by helping to identify disease pathogenesis.
\end{abstract}

Keywords: AITD, SLE, TNFSF4, Bioinformatics, in silico

\section{Introduction}

TNFSF4 (Tumor Necrosis Factor Ligand Superfamily Member 4) is a gene which encodes cytokines and members of this family are expressed on $\mathrm{T}$ cells, several of which are upregulated chasing T- cell activation [1]. The precursor cell source of TNFSF is macrophages, natural killer (NK) cells and $\mathrm{T}$ cells that occurs a mainly cellular targets and biological effects such as inflammation and coagulation in endothelial cells, activation in neutrophils, fever in hypothalamus, catabolism in muscle and fat [1-3]. TNFSF members characteristically form homotrimers, either within the plasma membrane or after proteolytic release from the membrane, and attach to homotrimeric TNF receptor superfamily (TNFRSF) molecules, which then start an assortment of signaling pathways [4]. TNFSF4 plays significant role in many diseases as atherosclerosis [5], allergic rhinitis, asthma [6], Vogt Koyanagi Harada disease (VKH), Behcet's diseases [7] and systemic lupus erythematosus (SLE) [8] and so it can also be correlated with other autoimmune diseases. Single nucleotide polymorphisms (SNPs) studies can have huge outcomes of the increasing risk of various widespread diseases. 
Some diseases strongly affect people depending on TNFSF. One of these diseases is AITDs and the other one is SLE. Autoimmune thyroid disease (AITD), mainly consists of Graves' disease (GD) and Hashimoto's thyroiditis (HT) [9]. AITD affects approximately 5\% of the general population and the prevalence of antithyroid antibodies is greater in females. According to National Health and Nutrition Examination Survey (NHANES III) study, hypothyroidism was found in $4.6 \%$ of the U.S. population $(0.3 \%$ clinical and $4.3 \%$ subclinical $)$ and hyperthyroidism in $1.3 \% \quad(0.5 \%$ clinical and $0.7 \%$ subclinical $)$ making autoimmune thyroid disease related thyroid dysfunction one of the most common diseases for the population [10]. If we consider autoimmunity to the thyroid (the presence of antibodies to thyroid antigens), it is as high as $10-20 \%$ of all women [11]. AITDs is organ specific disease and also the most common $\mathrm{AD}$ and is affiliated with other organ-specific and non-organ specific ADs.

SLE is an autoimmune disorder that has an effect on mainly women of childbearing age. Systemic lupus erythematosus (SLE) is also a multi-organ disorder that presents itself in a thousand ways. Since these patients have self-reactive B cells that are abnormally regulated, it can cause several disorders, including multisystem organ involvement, autoantibody production, immune complex deposition and complement activation with tissue damage. In other words, SLE can mimic many diseases. Its clinical course is extremely unpredictable, which makes diagnosis and treatment a challenge for clinicians. SLE is unique among other autoimmune disorders, because as well as having an unpredictable clinical course, it can directly affect any organ [12]. It appears that the clinical course of SLE is determined by genetic material in combination with environmental factors like in AITD. The incidence of SLE is between 0.3 and 23.2 in 100,000 person-years varying between populations [13].

Our study explains the relationship between AITD and SLE as in silico. Therefore; SNPs are shown with an explanation as deleterious or tolerant. Some specific and significant programs as SIFT, PolyPhen, SNP Mining, GeneMania, Exome Variant Server and UTRs Scan Programme are used for detection of input gene that identifies its homology. Recently, approximately 5,000,000 variants were analyzed in the coding region of human population [9]. The susceptibility SNPs for TNFSF4 gene haven't been conjecturable to date in silico study. In silico models allows scientists to experiment design and select a compound with a reasonable potential for further development of studies. Moreover; in silico platform can strongly lead the evolution of clinical biomarkers and preclinical assays to identify patient types and drug combinations, thus it can present information in SNPs studies to find out human complex disorders. The purpose of this study is to find out polymorphisms in TNFSF4 and to show which SNPs are linked to SLE and AITD.

\section{Materials and Method}

\subsection{GeneMANIA Algorithm for Functionally Similar Genes}

Gene Mania is a database which shows similar genes. It is used for functionally similar to input gene that is identified physical interactions, co- expression, co-localization, genetic interactions. It gives a query list by using genomics and proteomics data. This data is available at https://genemania.org [10] This data was used for TNFSF4 and its relations with other genes, thus it was predicted the function of TNFSF4 gene and gene set.

\subsection{Predicting Functional, Deleterious and Tolerated SNPS Prediction}

Sorting Intolerant from Tolerant Data (SIFT) is an online database that was used to search analogue sequences and select likely associated sequences which have similar function to input gene. There are some important points. According to SIFT, if the positions with standardized possibilities are lower than 0.05 , these are called as deleterious but if the positions with standardized possibilities are better than or equal to 0.05 , these are called as tolerated. This data is available at http://sift.jcvi.org [11].

\subsection{Exome Variant Server}

Exome Variant Server is procured by the The National Heart, Lung and Blood Institute (NHLBI). The purpose of this database is to find out novel genes and mechanisms accession to heart, lung and blood disorders. This database is available at http://evs.gs.washington.edu/EVS/ [12]. This database was used to determine the number of variations according to population, then the alleles were listed. EA (European African Population) allele counts and AA (African American population) allele counts was observed. As a result, the minor-allele frequency was analyzed in this data. AA, EA genotypes were calculated and evaluated.

\subsection{UTRscan Programme}

UTRscan is a database which is used for pattern matcher. It is used to investigate DNA, RNA, tRNA sequences that aims to get UTR motifs and these motifs are symbolical of 3'UTR and 5'UTR sequences. This is an online database that is used for collection of purposive sequence patterns placed in the 5'- or 3'-UTR sequences. The database is available at http://utrdb.ba.itb. cnr.it/ [13]. Some biological processed were analyzed by UTR scan such as determination, transcriptional regulatory pathways, and translational efficiency. 


\section{Results}

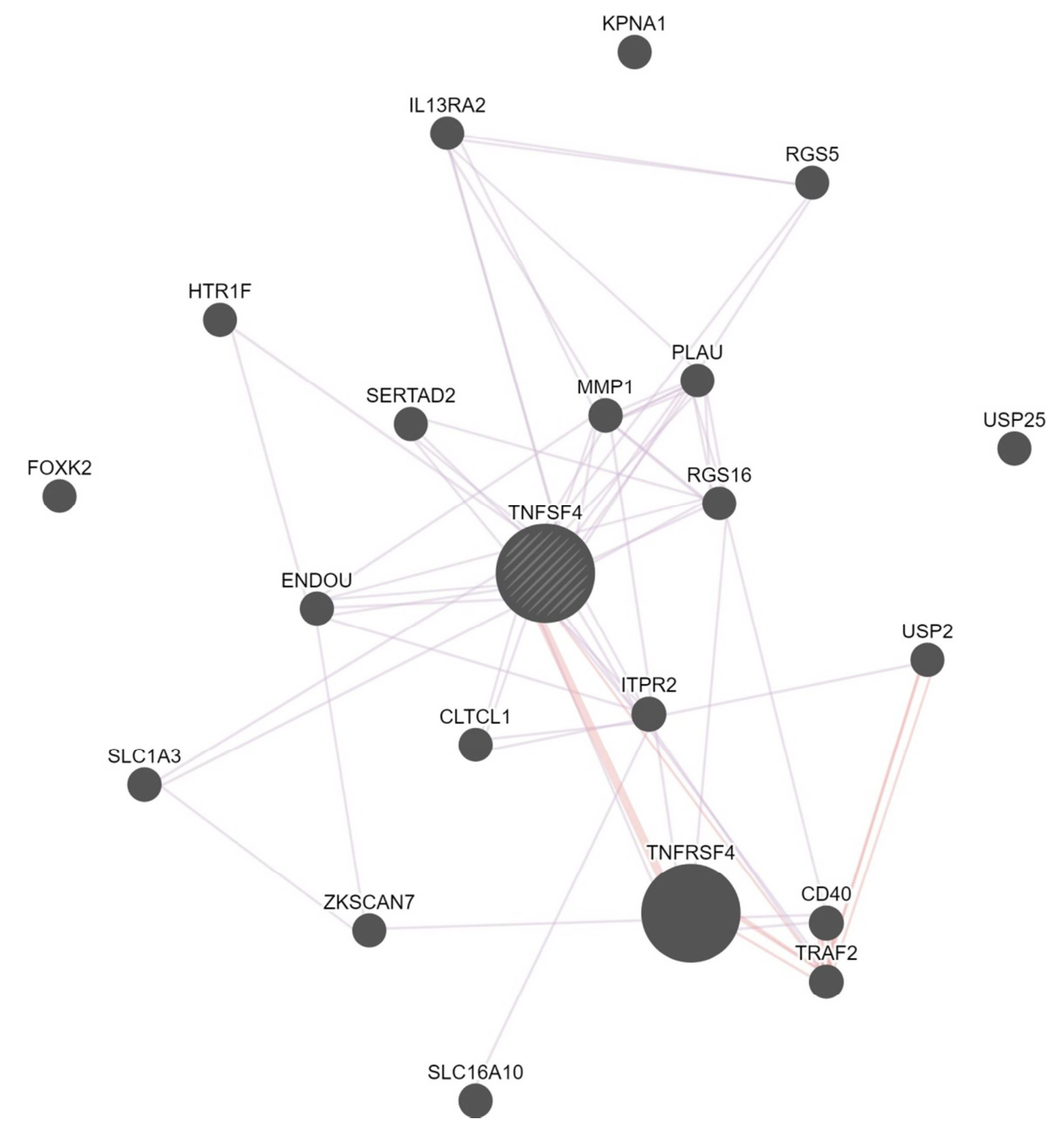

Figure 1. The picture shows that physical interactions (pink) and co-expression (lilac) between TNFSF4 gene and its related genes.

Table 1. The data descriptions of genes and their co-expressions and physical interactions with TNFSF4 gene network.

\begin{tabular}{lllll}
\hline Number & Gene's Name & Explanation & Co-expression & Physical Interaction \\
\hline 1 & HTR1F & 5-hydroxytryptamine receptor 1F & YES & NO \\
2 & FOXK2 & Forkhead box K2 & NO & NO \\
3 & SLC1A3 & Solute carrier family 1 member 3 & YES & NO \\
4 & ENDOU & Endonuclease poly (U) spesific & YES & NO \\
5 & SERTAD2 & SERTA domain containing 2 & YES & NO \\
6 & ITPR2 & Inositol 1,4,5-triphosphate receptor type 2 & YES \\
7 & TNFSFR4 & Tumor necrosis factor super family receptor 4 & NO & YES \\
8 & UPS2 & Ubiquitin spesific peptidase 2 & NO & NO \\
9 & RGS5 & Regulator of G protein signaling 5 & YES & YES \\
10 & CD40 & CD 40 molecule & NES & NO \\
11 & TRAF2 & TNF receptor associated factor 2 & NO \\
12 & USP25 & Ubiquitin spesific peptidase 25 & YES & NO \\
13 & PLAU & Plasminogen activator, urokinase & NO & NO \\
14 & SLC16A10 & Solute carrier family 16 member 10 & NO & NO \\
15 & KPNA1 & Karyopherin subunit alpha 1 & YES & NO \\
16 & MMP1 & Matrix metallopeptidase 1 & YES & NO \\
17 & IL12RA2 & Interleukin 13 receptor subunit alpha 2 & YES & NO \\
18 & CLTCL1 & Clathrin heavy chain like 1 & NO & \\
19 & ZKSAN7 & Zinc finger with KRAB and SCAN domains 7 & YES & \\
20 & RGS16 & Regulator of G-protein signaling 16 & \\
\hline
\end{tabular}

Table 1 shows the co-expression and physical interaction with TNFSF4 gene network. We explained twenty different genes and one of them is very significant gene which is known as CD40 molecule. Four genes (SLC16A10, KPNA1, 2KASANA, FOXK2) which have no interaction and co-expression with TNFSF4 were also found. 
Table 2. This data explains that TNFSF4 network genes' functions and its genome.

\begin{tabular}{llll}
\hline Function & False Discovery Rate & Genes in network & Genes in genome \\
\hline Regulation of production of molecular mediator of immune response & 0.2680558227214164 & 3 & 44 \\
Regulation of t cell cytokine production & 0.3188879419816194 & 2 & 13 \\
Production of molecular mediator of immune response & 0.31888794198161943 & 3 & 69 \\
Positive regulation of production of molecular mediator of immune response & 0.31888794198161942 & 2 & 16 \\
T cell cytokine production & 0.31888794198161942 & 2 & 16 \\
Regulation of immunoglobulin production & 0.31888794198161942 & 2 & 16 \\
Positive regulation of cytokine production involved in immune response & 0.31888794198161942 & 2 & 13 \\
CD40 receptor complex & 0.31888794198161942 & 2 & 11 \\
B cell activation & 0.46276942084836675 & 3 & 119 \\
Positive regulation of adaptive immune response & 0.46276942084836675 & 2 & 34 \\
\hline
\end{tabular}

A major key role of UTRs is to control mRNA stability, turnover, and translation effectiveness and in this study; UTRscan data gave 46 different genes. If various sequences for every UTR SNP are discovered to have various functional patterns, this UTR SNP is guessed to have functional meaning that this study has correlated this situation.

Table 3. SNP list was provided for functionally important by SIFT data.

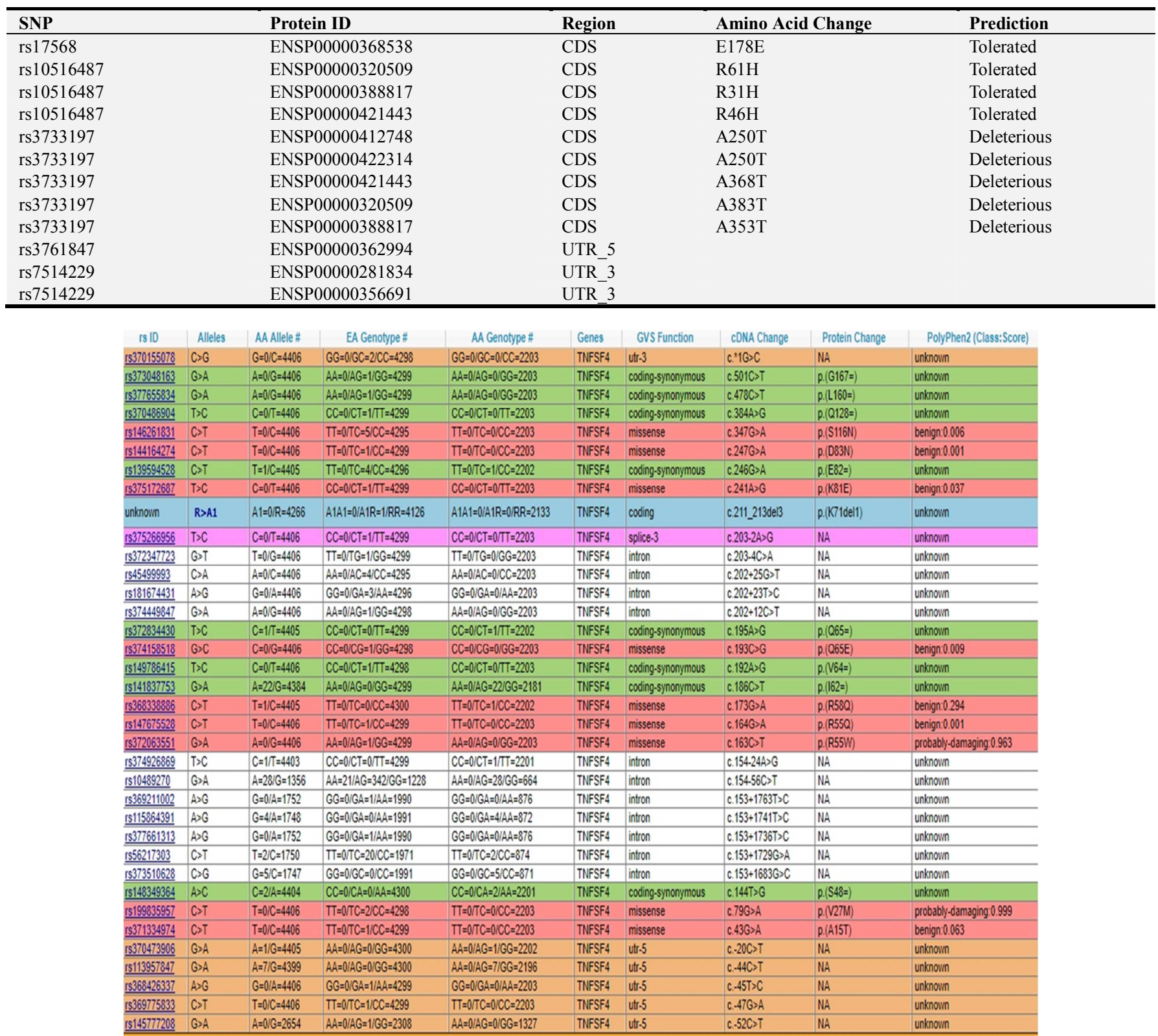

Figure 2. This data shows SNPs of TNFSF genes that identifies splice or nonsense or frameshift (pink), missense (red), coding synonymous (green), coding (blue), utr (orange), coding complex (yellow) by NHLBI Exome Sequencing Project. 
Our results showed the polymorphism changes of African American and European African population (Figure 2). We have determined 8 coding-synomymous, 9 missense, 1 coding, 1 splice-3, 1 utr-3, 5 utr-5 and 11 intron variation in CD40 molecule. Two of them could be damaging mutation for humans.

\section{Discussion}

Most recently, studies showed that polymorphisms and expressions of TNF superfamily cytokines, receptors, indicating proteins and genetic variations are related to autoimmune, autoinflammatory and inflammatory diseases. However, this situation has continued to be explored [14]. Some polymorphisms have exposed direct results on gene expression and infrequently downstream phenotype for illness-related variants positioned TNFSF4.

In this study, the CD40 was found very close to TNFSF4. Because; CD40 belongs to the TNF receptor family that its ligand, CD40L (CD154), is a trimeric membrane protein that is homologous to TNF and it is expressed on B cells. One of the major indicators for activation is IFN- $\gamma$ from Th1 type CD4 $\mathrm{T}$ cells in the macrophage and the other indicator is CD40L on the T cell that combined CD40 on the macrophage cell surface. Consequently, the macrophage expresses much more CD40 and TNF receptors on its surface that helps raise the level of activation.

According to the results, GeneMania shows that CD40 gene has physical interaction and co-expression with TNFSF4 and TNFSF gene. TNFSFR are belong to structurally homologous transmembrane proteins which attach TNFSF proteins and produce signals that organize proliferation, differentiation, apoptosis, and inflammatory gene expression thus it can cause autoimmune diseases. HTR1F, FOXK2, SLC1A3, ENDOU, SERTAD2, ITPR2, RGS5, PLAU, MMP1, IL12RA2, CLTCL1, RGS16 genes have co-expression with TNFSF4 that explains synchronical recognition, clustering and investigation of thousands of genes with analogous patterns across many forms [15]. TRAP2, MMP1, IL12RA2 genes have been studied with SLE $[16,17]$, autoimmune diseases [14, 18] and thyroid [19] diseases but there aren't any studies on SLE, autoimmune diseases, and thyroid diseases with other genes. The TNFSF4-associated genes' functions and its appearance in the genome were grouped that defines as if it is greater than or equal to the probability that this is a false positive.

SIFT data was shown that rs3733197 had deleterious and rs10516487, rs17568 had tolerated amino acid changes, thus this situation is also very important because of its effects on protein's functionality. According to Figure 2, there were discovered 37 variants in European American and African American population. 9 missense, 8 coding synonymous, 1 coding, 1 splice-3,1 utr-3, 11 intron, 5 utr-5 variants were analyzed that two of them SNPs that these are rs199835957, rs372063551 were shown probably damaging by PolyPhen2 and 13 unknown SNPs was described as in silico. There was no information about 37 variants and there haven't been reported in ClinVar before, so there haven't shown any clinical link with diseases. Our findings demonstrated that harmful (damaging) SNPs in the TNFSF4 gene can be the cause or causes of problems and they can be significant candidate for various types of human diseases related to TNFSF4.

In the future, genes of TNFSF4, TNFSFR and CD40 may be studied on polymorphisms with experimental analysis in order to contribute to identify disease pathogenesis. Therefore, our study is promising for the development of more effective drug design.

\section{Conclusion}

TNFSF4 is a gene that encodes cytokines and members of this family are expressed on $\mathrm{T}$ cells, several of which are upregulated chasing $\mathrm{T}$ - cell activation. Therefore, TNFSF4 can play significant role in inflammatory diseases that its polymorphisms of the TNFSF4 gene are mostly related with Sjogren's syndrome, and systemic lupus erythematosus (SLE). In this study, genetic variations that may alter the expression, function and role of TNFSF4 were investigated by using bioinformatics tools. Our findings demonstrated that harmful (damaging) SNPs in the TNFSF4 gene can be responsible of abnormalities and they can be significant candidate to be the cause of various types of human diseases related to TNFSF4.

\section{References}

[1] Croft M. The Role of TNF Superfamily Members in T-cell Function and Diseases. Nat Rev Immunol. 9 (4): pp. 271-85.

[2] Ward KL, Lin WW, Sedy JS, Ware CF. The TNF Receptor Superfamily in Costimulating and Coinhibitory Responses. Immunity. 44 (5): pp. 1005-1019. 2016.

[3] Croft M. et al. TNF Superfamily in Inflammatory Disease: Translating Basic Insights. Trends Immunol. 33 (3): pp. 14452. 2012.

[4] Sonar S, Lal G. Role of Tumor Necrosis Factor Superfamily in Neuroinflammation and Autoimmunity. Front Immunol. 6: pp. 364. 2015.

[5] Cheng J. et al. Association between TNFSF4 tagSNPs and Myocardial Infarction in a Chinese Han Population. Genet. Mol. Res. 14 (2): pp. 6136-6145. 2015.

[6] Liu Y. et al. Genetic Risk of TNFSF4 and FAM167A-BLK Polymorphisms in Children with Asthma and Allergic Rhinitis in a Han Chinese Population. Journal of Asthma. 53 (6): pp. 567-575. 2016.

[7] Lu S. et al. Association of TNFSF4 Polymorphisms with Vogt-Koyanagi-Harada and Behcet's Disease in Han Chinese. Sci Rep, 6. 2016.

[8] Gupta V. et al. Association of ITGAM, TNFSF4, TNFAIP3 and STAT4 Gene Polymorphisms with Risk of Systemic Lupus Erythematosus in a North Indian Population. Lupus, 27 (12): pp. 1973-1979. 2018. 
[9] Brix TH, Hegedus L. Twin Studies as a Model for Exploring the Aetiology of Autoimmune Thyroid Disease. Clin Endocrinol (Oxf). pp. 76 (4): 457-64. 2012.

[10] Hollowell JG. et. al. Serum TSH, T (4), and Thyroid Antibodies in the United States Population (1988 to 1994): National Health and Nutrition Examination Survey (NHANES III). J Clin Endocrinol Metab. 87 (2): 489-99. 2002.

[11] Tomer Y. Mechanisms of Autoimmune Thyroid Diseases: from Genetics to Epigenetics, Annu. Rev. Pathol. 9 147e156. 2014.

[12] Javinani A, Ganjouei AA, Aslani S, Jamshidi A, Mahmoudi M. Exploring the Etiopathogenesis of Systemic Lupus Erythematosus: a Genetic Perspective. Immunogenetics. 2019.

[13] Rees F, Doherty M, Grainge MJ, Lanyon P, Zhang W, The Worldwide Incidence and Prevalence of Systemic Lupus Erythematosus: a Systematic Review of Epidemiological Studies. Rheumatology (Oxford) 56: 1945-1961. 2017.

[14] Rajasekaran R. et al. Computational and Structural Investigation of Deleterious Functional SNPs in Breast Cancer BRCA2 Gene. Chinese Journal of Biotechnology. 24( 5): pp. 851-6. 2008.

[15] Montojo J. et al. GeneMANIA: Fast Gene Network Construction and Function Prediction for Cytoscape. F1000Res. 3: pp. 153. 2014.

[16] Ng PC, Henikoff S. Predicting Deleterious Amino acid Substitutions. Genome Res. 11 (5): p. 863-74. 2001.

[17] Ng PC, Henikoff S. SIFT: Predicting Amino acid Changes that Affect Protein Function. Nucleic Acids Res. 31 (13): p. 38123814. 2003.
[18] Ng PC, Henikoff S. Predicting the Effects of Amino acid Substitutions on Protein Function. Annual Review of Genomics and Human Genetics. 7: p. 61-80. 2006.

[19] Richard AC. et al. Targeted Genomic Analysis Reveals Widespread Autoimmune Disease Association with Regulatory Variants in the TNF Superfamily Cytokine Signalling Network. Genome Med. 2016.

[20] Serin EAR. et al. Learning from Co-expression Networks: Possibilities and Challenges. Frontiers in Plant Science, 2016.

[21] Zhu LJ. et al. Altered Expression of TNF-alpha Signaling Pathway Proteins in Systemic Lupus Erythematosus. Journal of Rheumatology. 37 (8): p. 1658-1666. 2010.

[22] Weinberger P. et al. Cell Cycle M-Phase Genes Are Highly Upregulated in Anaplastic Thyroid Carcinoma. Thyroid. 27 (2): p. 236-252. 2017.

[23] Leonardi A. et al. Endoplasmic Reticulum Stress Causes Thyroglobulin Retention in This Organelle and Triggers Activation of Nuclear Factor-kappa B via Tumor Necrosis Factor Receptor-associated factor 2. Endocrinology. 143 (6): p. 2169-2177. 2002.

[24] Wigren M. et al. Cardiovascular Disease in Systemic Lupus Erythematosus is Associated with Increased Levels of Biomarkers Reflecting Receptor-activated Apoptosis. Atherosclerosis. 270: p. 1-7. 2018.

[25] Kutukculer N. et al. CD4+CD25+Foxp3+ T regulatory cells, Th1 (CCR5, IL-2, IFN-gamma) and Th2 (CCR4, IL-4, Il-13) Type Chemokine Receptors and Intracellular Cytokines in Children with Common Variable Immunodeficiency. Int J Immunopathol Pharmacol. 29 (2): p. 241-51. 2016. 